\title{
Research Article \\ Effect of Height on Pedestrian Route Choice between Stairs and Escalator
}

\author{
Qian Li, ${ }^{1}$ Changxu Ji, ${ }^{2}$ Limin Jia, ${ }^{1,2}$ and Yong Qin ${ }^{1}$ \\ ${ }^{1}$ School of Traffic and Transportation, Beijing Jiaotong University, Beijing 100044, China \\ ${ }^{2}$ State Key Laboratory of Rail Traffic Control and Safety, Beijing Jiaotong University, Beijing 100044, China
}

Correspondence should be addressed to Limin Jia; jialm@vip.sina.com

Received 18 November 2013; Revised 22 January 2014; Accepted 10 February 2014; Published 13 March 2014

Academic Editor: Wuhong Wang

Copyright (C) 2014 Qian Li et al. This is an open access article distributed under the Creative Commons Attribution License, which permits unrestricted use, distribution, and reproduction in any medium, provided the original work is properly cited.

In order to overcome the subjectivity of existing pedestrian route choice models, an alternative choice model is presented based on the utility equation. It is composed of several indirectly objective characteristic variables, including the height, length, and width of interlayer facilities; speed of automated facilities; and carry-on luggage. Considering the scene that pedestrians choose between the stairs or escalators, an extended binary logit model is developed. Calibration and validation of the model are accomplished by using the data collected in four typical passenger transfer stations in Beijing, China. The results show that the proposed model has an average accuracy of $86.56 \%$ in bidirection for predicting pedestrians' behavior. An interesting phenomenon can be found that the length of facility has poorer impact than height on pedestrians' route choice behavior. Some quantitative and irradiative conclusions have been illustrated on the relationship between the selection probability and the variables, which is expected to be valuable for extracting the implicit theoretical mechanism of passenger choice behavior.

\section{Introduction}

Integrated development of the modern passenger transfer hubs realizes the combination of intercity and in city transportation within or in the same space layout. Centralized transferring of pedestrians greatly improve the operating efficiency of the urban passenger transport network. Passengers transfer space has a multilayer and attaches a certain amount of interlayer facilities connection, such as stairs, escalators, elevators and slopes. The three-dimensional structure increases the complexity of the traveler's behavior and activities in the hub. At peak time, due to the impact of a large number of passengers, bottlenecks usually appear at interlayer facilities, which induces local congestions and unnecessary delays. Therefore, understanding of the role of the relationship between these facilities and pedestrian behavior has become a very compelling issue [1-4], as the dynamic interactions of person-to-person $[5,6]$ and vehicleto-vehicle [7]. Although these problems are very specific, they also implied considerable theoretical mechanism. Mastering this knowledge can help building designers to design more reasonable structure of facilities, make pedestrians use the facilities relatively balanced, promote the improvement of critical facilities' level of service [4], and finally let pedestrians get enjoyable experience in the process of travel. In order to achieve this goal, it becomes very important to define the characteristics of passengers' choice behavior among interlayer facilities, and to estimate the effect of physical properties of interlayer facilities on the choice behavior.

As is known to all, in the process of pedestrians through level bridging facilities, the physical exertion must be paid by the tourists that have high-positive correlation with the height of facilities, which plays a key role in their choice decisions, especially those carrying luggage. Moreover, this must be related to the fundamental structure of facilities and current environmental factors such as flow density. There are lots of researches on modeling and analyzing the passenger choice behavior of facilities. Daamen et al. [3] built utility analysis model for selection problem of the entire path of the longitudinal facilities. Seneviratne and Morrall [8] and Guo et al. [9] analyzed detailed influencing factors of pedestrian route choice behavior. Borgers and 
Timmermans [10] established the selection model of shortest walking distance in horizontal dimension. Cheung and Lam [11] took pedestrians in Hongkong as an example, studied the passenger facilities choice behavior, and proposed the perceive time as an influence factor. Ye et al. [12] analyzed the traffic characteristics of the passenger of stairs and corridors in the Shanghai subway station and established the relationship between the interlayer facilities and the volume of passenger traffic. Through researching the relationship between the interlayer facilities layout and passengers' route choice in the transfer station, such as stairs and escalators, Daamen [1] and Zeiler et al. [13] summed up the degree of passenger congestion of interlayer facilities as a key factor directly impact on behavior of passengers. Jia et al. [14] based on comprehensive analysis characteristics of the passenger flow and service status of the inner facilities in the hub established a systematic analysis method of capacity adaptability of facilities' ability. Lam and Chau [15] according to the variation of pedestrian flow of shopping center in station developed multiple walking facilities control strategy to reduce resource waste. In addition, Olander and Eves [16] and Lewis and Eves [17] analyzed the influence of choice-onpoint prompt on enhancing the use of stairs by pedestrians, from the perspective of human health.

In short, these studies have focused on the relationship between passenger flow and interlayer facilities to reveal the theoretical mechanism of passenger choice behavior, ignoring the effect of interlayer facilities physical properties on passengers. As for the models, discrete utility theory has been extensively used on these issues, whether in horizontal or vertical direction. The difference between these models is the selection of influencing factors on calculation utility function, such as the generalized consumption [3], shortest walking distance [10], and generalization of time [11]. All of these did not positively consider the height of facilities which is a quantitatively specific parameter. Take the perception time, for example, since it is a highly technical matter for people to reasonably map the objective existence of physical exertion paid by climbing stairs to the subjective perception time, even if the professional architect also hardly completes the transform, the results of theoretical analysis in the application of engineering practice are at stake.

On the basis of previous research, the height of facilities was proposed as an independent factor in a utility model to describe the relationship between height of facilities and pedestrian facilities choice behavior.

After a brief literature review, the new model was put forward, and some of the features were expounded in detail. Then, the scene of the traffic data which model calibration required was introduced, and the model calibration results were shown. Finally, theoretical characteristics were illustrated which extracted from further analysis of the model and came to some meaningful conclusions.

\section{Model and Calibration}

2.1. Analysis of Influence Factors. To calculate the utility function, we need select the influencing factors. These is the most fundamental problem. An extended literature study on empirical data and modeling of pedestrian route choice can be found in $[1,3,8]$. Generally, three sets of characteristics would be taken into concern, including the facility structure, basic consumption, and passenger property.

(i) Facility Structure. The number of routes, one of the most important factors, is related to the categories of the mathematical model. Length of facility is closely related to the time which passenger need to get through the facility, also it is a primary factor to analyze the choice behavior and more important in the pedestrian route choice behavior model in horizontal dimension [10].

(ii) Basic Consumption. Time consumption at least consists of two parts, including the travel time and waiting time, which are under the influence of the length of facilities, passenger current speed, channel capacity, and the number of waiting for lineup.

Passengers will take the corresponding physical strength when they are using level bridging facilities. Within reasonable delay time, people tend to choose less expensive physical facilities. That is to say, physical consumption plays a substantial role in pedestrians' choice in vertical dimension. If passengers carrying luggage (we consider only the heavy luggage without light handbag), more energy will be consumed when passengers go through the non-automated facilities.

(iii) Passenger Attribute [3, 8]. The effects of differences in gender and age of passengers are not obvious. Also, trip purpose plays no role for work-related walking trips. This paper focuses on the ordinary pedestrians without difference of purpose.

After an overview of the influencing factors above, the structure and basic consumption are selected for subsequent modeling; passenger property will not be considered. Even, the items of data collection are corresponding with them.

2.2. Binary Logit Model. Theory of disaggregate model is based on the hypothesis that consumers choose to pursue utility maximization. In the issue of the passenger facilities selection, passenger choice behavior and consumer have the same principle. Logit model, which has advantages of simple structure and strong applicability, divides utility into uncertainty utility and random utility and assumes that the random utility obeys certain probability distribution, thus obtains the probability of travelers choosing different transport facilities.

Assume that the passengers can select the options independently, the utility model $U_{\text {in }}$ of the passenger $n$ selection facilities $i$ can be expressed as

$$
U_{i n}=V_{i n}+\varepsilon_{i n}
$$

where $V_{i n}$ is the fixed term in utility function of traveler $n$ that selects facility $i$ and $\varepsilon_{\text {in }}$ is the random term in utility function of traveler $n$ that selects facility $i$.

Constructing and evaluating the utility function is a key link in the process of correctly analyzing passenger choice behavior. Borgers and Timmermans [10] formulate a model for pedestrian route choice in city centers given pedestrian's 
TABLE 1: Characteristic variables.

\begin{tabular}{lcc}
\hline \multirow{2}{*}{ Characteristic variables } & \multicolumn{2}{c}{ Variables represent } \\
& Escalator & Stairs \\
\hline Time consumption & $t_{1}^{\text {esc }}$ & $t_{1}^{\text {st }}$ \\
Walking time $\left(t_{1}\right)$ & $t_{2}^{\text {esc }}$ & $t_{2}^{\text {st }}$ \\
Delay time $\left(t_{2}\right)$ & & \\
Physical consumption & 0 & $h^{\text {st }}$ \\
Height $(h)$ & 0 & $g^{\text {st }}$ \\
Luggage $(g)$ &
\end{tabular}

destination. It is assumed that route choice is primarily influenced by the distance of the alternative routes. Cheung and Lam [11] adopt a logit model to model pedestrian's choice between escalators and stairs in a station, in which the utility of an alternative $U_{r}$ is described by

$$
\begin{aligned}
& U_{r}=V_{r}+\varepsilon_{r} \\
& \text { with, } V_{r}=\alpha+\beta T_{r} \\
& \text { with, } T_{r}=t_{r \text {,delay }}+t_{r, \text { walk }},
\end{aligned}
$$

where $\alpha$ and $\beta$ are parameters to be estimated, $T_{r}$ is the travel time along route $r, t_{r \text {,delay }}$ is delay on the walkway leading to escalator or stair, $t_{r \text {,walk }}$ is the travel time along escalator or stair. Rewriting the resulting logit model leads to the following expressions:

$$
\begin{gathered}
P_{\text {st }}=\frac{\exp \left(V_{\text {st }}\right)}{\exp \left(V_{\text {st }}\right)+\exp \left(V_{\text {esc }}\right)}=\frac{1}{1+\exp \left(V_{\text {esc }}-V_{\text {st }}\right)} \\
P_{\text {esc }}=1-P_{\text {st }},
\end{gathered}
$$

where $P_{\text {st }}$ is the probability of pedestrian choosing escalator, $P_{\text {esc }}$ is the probability of pedestrian choosing stairs, and $\left(V_{\text {esc }}-\right.$ $\left.V_{\text {st }}\right)$ is relative discomfort measure for using stairs.

Combining with the characteristics of passenger choice behavior in vertical dimension, the time and physical consumption are select and used as characteristic variables, as shown in Table 1. Time consumption consists of walking time $t_{1}$ and delay time $t_{2}$. Physical consumption consists of interlayer height $h$ and carry-on luggage $g$. In this work, passengers within automated facilities are regard as having the physical consumption as zero, and only distinguish whether passengers carrying luggage or not. 0 for nonluggage, otherwise is 1 .

Then, the new $V_{r}$ is replaced with

$$
V_{r}=\theta_{0}+\theta_{1}\left(t_{1}+t_{2}\right)+\theta_{2}(h+g) \text {, }
$$

where $\theta_{0}, \theta_{1}$, and $\theta_{2}$ are parameters to be estimated.
Set

$$
\begin{aligned}
V_{\mathrm{esc}} & -V_{\mathrm{st}}=\theta_{0}+\theta_{1} \Delta T+\theta_{2} \Delta P \\
\Delta T & =\left\{\left(t_{1}^{\mathrm{esc}}+t_{2}^{\mathrm{esc}}\right)-\left(t_{1}^{\mathrm{st}}+t_{2}^{\mathrm{st}}\right)\right\} \\
& =\left\{\left(t_{1}^{\mathrm{esc}}-t_{1}^{\mathrm{st}}\right)+\left(t_{2}^{\mathrm{esc}}-t_{2}^{\mathrm{st}}\right)\right\} \\
& =\Delta t_{1}+\Delta t_{2} \\
\Delta P & =\left\{\left(h^{\mathrm{esc}}+g^{\mathrm{esc}}\right)-\left(h^{\mathrm{st}}+g^{\mathrm{st}}\right)\right\} \\
& =-\left(h^{\mathrm{st}}+g^{\mathrm{st}}\right) .
\end{aligned}
$$

Substituting (5) into (3), (6) will be available as

$$
\begin{gathered}
P_{\mathrm{esc}}=\frac{1}{1+\exp \left(\theta_{0}+\theta_{1} \Delta T_{1}+\theta_{2} \Delta T_{2}+\theta_{3} h^{\mathrm{st}}+\theta_{4} g^{\mathrm{st}}\right)} \\
P_{\mathrm{st}}=1-P_{\mathrm{esc}} .
\end{gathered}
$$

\subsection{Model Estimation}

2.3.1. Basic Data. To exclude the interference of other transportation modes and obtain pedestrian traffic flow parameters in a range as wide as possible, four transfer stations were selected as the observation sites in Beijing, such as Xizhimen subway station, Beijing South Railway Station, Zoo Station, and Zhichun Road Station, for they have large amounts of vertical pedestrian travel demand. And, the number of different choice situations and route alternatives is reasonably high in these stations.

The way of basic data acquisition is video observations, as described by Cheung and lam [11]. The pedestrian flows were recorded with Digital Camera for an hour, respectively, during the peak times and the information in the video were extracted manually and organized in the form of individual cases afterward. 3849 passengers' choice decisions, 2207 in ascending and 1642 in descending, had been extracted from 40 video files of 10 scenes allocated in the four stations. Some snapshots can be seen in Figure 1. The data has been split into two groups, one is for calibrating and the other for validating.

The factors considered as well as the corresponding data obtained from the videos in the study include date, peak time, station, direction, structure of facilities (height, length, and width), speed of automated facilities, the number of people in queue for escalator, and whether carry-on luggage.

2.3.2. Model Calibration. Model calibration is divided into three steps. Firstly, the form of the utility function and the characteristic variable must be determined, on behalf of calibration of the model. Secondly, using the maximum likelihood estimation method to calibrate the parameters and making some preparations for model validations. Finally, the covariance matrix is used to make $t$ test or other tests. The results of model test are shown in Table 2 .

As the absolute value of the $t$-test is larger than 1.96, there is 95 percentage degree of confidence on indicating that the corresponding variable is a factor that can affect the probability of selection. In Table 2, since the absolute values of 
TABLE 2: Calibration result.

\begin{tabular}{lcccccc}
\hline Direction & Type & $\theta_{0}$ & $\theta_{1}$ & $\theta_{2}$ & $\theta_{3}$ \\
\hline \multirow{2}{*}{ Ascending } & Value & 6.6324 & -0.2501 & -0.5986 & 0.8642 & 0.9976 \\
& $t$-test & 2.16 & 0.51 & 2.53 & -3.76 \\
\hline \multirow{2}{*}{ Descending } & Accuracy & $89.41 \%$ & Goodness & 0.86 & Conformity \\
& Value & 5.9077 & -0.5539 & -0.7086 & 0.7331 \\
\hline & t-test & 2.26 & -0.84 & -4.15 & 0.8244 \\
& Accuracy & $83.70 \%$ & Goodness & 0.79 & 2.17 \\
\hline
\end{tabular}

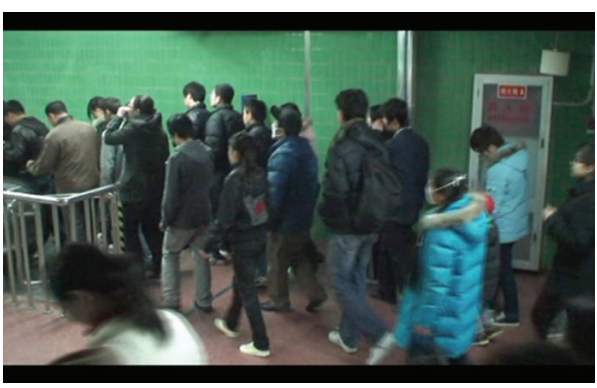

(a) In ascending

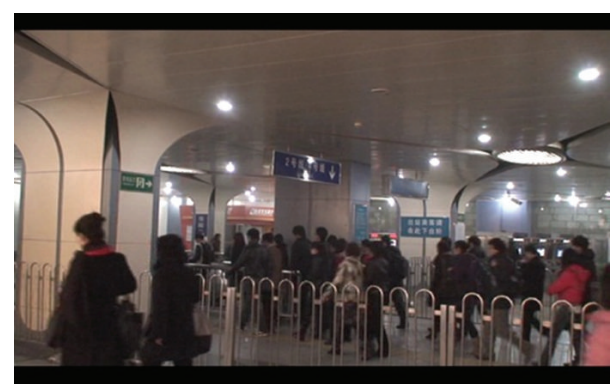

(b) In descending

FIGURE 1: Video snapshots.

$t$-test value on parameter $\theta_{1}$ are less than 1.96 in bidirections, the walking time $t_{1}$ is wiped off from the characteristic variables for its little affect. In terms of accuracy, the average accuracy of the models for forecasting the passengers' choice decisions is $86.56 \%$, which meets the precision requirements of BL model. The results of model calibration are as follows.

In ascending direction

$$
\begin{gathered}
P_{\text {st }}=\frac{1}{1+\exp \left(6.6324-0.5986 \Delta t_{2}+0.8642 h+0.9976 g\right)} \\
P_{\text {esc }}=1-P_{\text {st }} .
\end{gathered}
$$

In descending direction

$$
\begin{gathered}
P_{\mathrm{st}}=\frac{1}{1+\exp \left(5.9077-0.7086 \Delta t_{2}+0.7331 h+0.8244 g\right)} \\
P_{\mathrm{esc}}=1-P_{\mathrm{st}} .
\end{gathered}
$$

\section{Analysis of Choice Mechanism}

This section presents an examination of various properties of pedestrian choice behavior indicated in the percentages of the stairs that will be used. In line with $[3,11]$ the proposed model also showed that passengers are more inclined to choose escalators as they improve experience, more obvious in upward direction. For distinctively analyzing the performance of level bridging facilities, there are three factors that would be highlighted in the following, such as height of facilities, delay time, and carry-on luggage. A specific set of parameters to a passenger will produce a variety of results, which revealed some passenger's preferences on choice behavior.

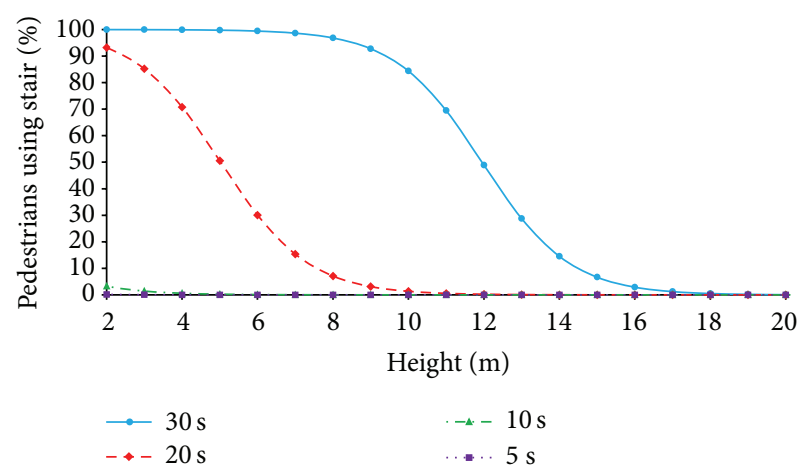

FIGURE 2: Percentage of pedestrians using stairs by different waiting time.

3.1. Varying Facilities Height. Figure 2 shows the percentage of pedestrians using stairs plotted against height of vertical access settings without luggage in ascending by delay time at $5,10,20$, and 30 seconds, respectively. Basically, with the passengers' delay time in front of escalator growing, the probability that they choose the stairs becomes higher.

When height is 5 meters and delay time is 20 seconds, the probabilities of each alternative are chosen almost the same. Once the balance is broken, passengers will more clearly to make a decision. In case of ignoring waiting time, the vast majority of people will not pay attention to the stairs. Escalator plays an important role in the process of transition between the layers. As the delay time changes from 10 to 20 seconds or more, the concern of pedestrians shows a dramatic shift, so much as more than 98 percentages of people choose stairs when the waiting time is for 30 seconds. Under the condition that the floor is not too high, 


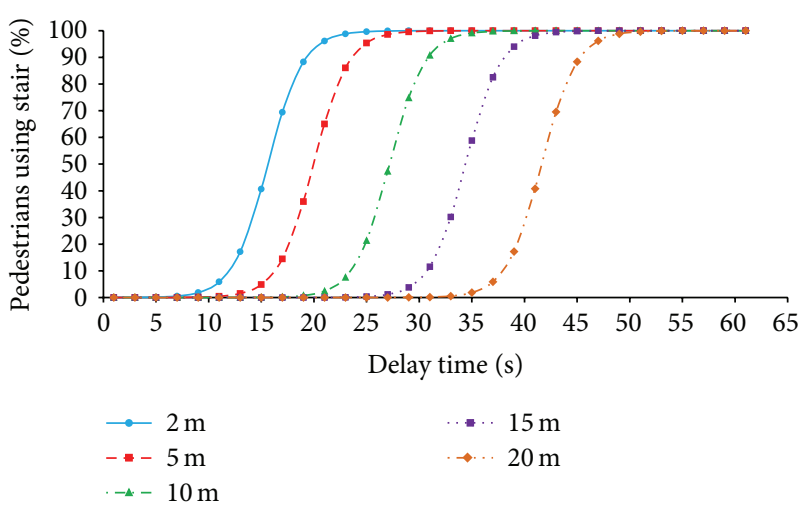

FIGURE 3: Percentage of pedestrians using stairs by different height.

it is necessary to appropriately increase the stairs width to encourage passengers to get through as soon as possible. Otherwise, a lot of passengers were forced to crowd in front of the facilities, although they are willing to pay extra strength in climbing the stairs.

3.2. Varying Delay Time. Figure 3 shows the percentage of pedestrians using stair plotted against delay time without luggage in ascending by height at $2,5,10,15$, and 20 meters, respectively. The height level bridging facility is larger, the passenger's acceptable delay time standing by escalator is longer, and the number of passengers in front of selector is more, under the selection probability of stairs is less than $15 \%$.

For example, as the height reaches 20 meters, the traveler's acceptable delay time will not exceed 35 seconds. Otherwise, a significantly increase occurs in probability of passengers choosing stairs. Then, a larger number of passengers will gather in the access corridor to the escalator.

In the design stage of infrastructures, corresponding to the height of the interlayer facilities, the area of access facilities to level bridging facilities must be large enough, at least for satisfying the demands of the corresponding number of passengers.

3.3. Carry-On Luggage or Not. Figure 4 shows the percentage of pedestrians using stairs against height of interlayer facilities with 20 seconds delay time in ascending, including the cases that they carry-on luggage or not, respectively. In these certain conditions, the percentage of choosing stairs in passenger with load is lower than lightweight ones within a certain range. As the interlayer height is 6 meters, the 53.85 percentage of passengers without luggage will take into account stair, and only 30.09 percent of passengers with luggage want to climb the stairs to avoid the delay time. Therefore, if the height of target facilities is more than 10 meters, almost all travelers tend to abandon the use of stair rationally, no matter whether they carry luggage or not. So, when the height reached a certain value, passengers' choice behavior will be convergence.

According to the analysis on effect of luggage to pedestrian's choice behavior, a good proposal could be put forward.

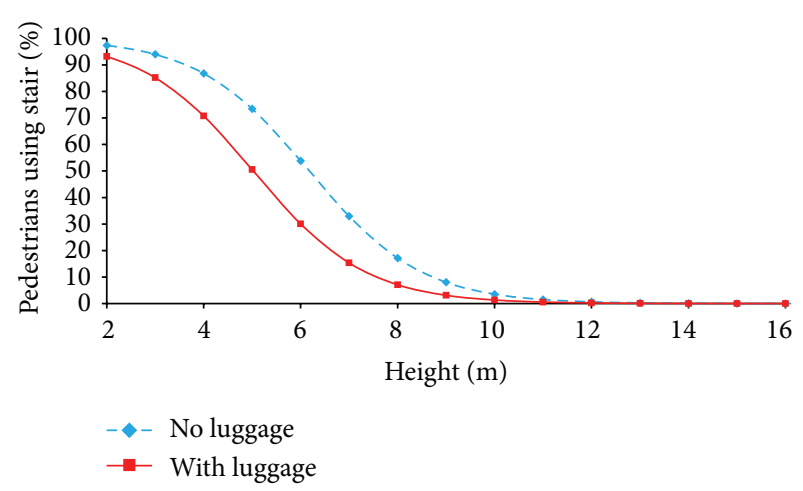

FIGURE 4: Effect of luggage on probability of passenger using stairs.

Compared to commuter staff, the proportion of intercity travelers which carrying luggage is higher and the number of escalator in intercity transportation hub should be more than transfer hubs in city, in order to improve the capacity of the automation facilities and provide better service to passengers.

\section{Conclusions}

This study developed a binary logit model with an alternative utility function to predict pedestrian's choice behavior between stair and escalator in public transfer stations. Different from the other models, the generalized extreme value indicated by subjective perception of time consumption of passenger uses level bridging facilities that have been divided into two parts. One is time consumption which consists of walking time and delay time, and the other is physical consumption which is represented by the height of facilities and whether carrying-on luggage. In addition, ascending and descending data for calibrating and testing the function has been collected in five passenger transfer stations in Beijing, China, including ten stairs and escalator parallel scenarios.

According to the results, the average accuracy of $86.56 \%$ is extraordinary high for this type of model. The model also shows that the length of facility has little influence on passenger selection behavior. So, it is reasonable that the height is identified as an independent characteristic variable. Some phenomena have been observed with further analysis of the model. Passengers are more sensitive to facilities height in ascending than in descending. Due to the objectivity of the selected parameter, we can intuitively understand the relationship between pedestrian choice behavior in vertical dimension and their specific environment. Also, the estimated values for infrastructure types may directly be applied in a simulation tool, even as a theoretical reference for facility design decisions.

\section{Conflict of Interests}

The authors declare that there is no conflict of interests regarding the publication of this paper. 


\section{Acknowledgments}

This work was supported by the Independent Research Topic of State Key Laboratory (RCS2011ZT002), National Nature Science Foundation of China (71171015), and National High Technology Research and Development Program (2011AA110506).

\section{References}

[1] W. Daamen, Modelling Passenger Flows in Public Transport Facilities, Delft University of Technology, Delft, The Netherlands, 2004.

[2] W. Daamen, P. H. L. Bovy, and S. P. Hoogendoorn, "Choices between stairs, escalators and ramps in stations," in Proceedings of the International Conference on Computer System Design and Operation in the Railway and Other Transit Systems, pp. 3-12, Prague, Czech Republic, July 2006.

[3] W. Daamen, P. H. L. Bovy, and S. P. Hoogendoorn, "Passenger route choice concerning level changes in railways stations," in Proceedings of the Transportation Research Board Annual Meeting, pp. 1-18, National Academy Press, Washington, DC, USA, 2005.

[4] Transportation Research Board, Highway Capacity Manual 2000, Transportation Research Board, Washington, DC, USA, 2000.

[5] D. Helbing, L. Buzna, A. Johansson, and T. Werner, "Selforganized pedestrian crowd dynamics: experiments, simulations, and design solutions," Transportation Science, vol. 39, no. 1, pp. 1-24, 2005.

[6] V. J. Blue and J. L. Adler, "Cellular automata microsimulation for modeling bi-directional pedestrian walkways," Transportation Research B, vol. 35, no. 3, pp. 293-312, 2001.

[7] W. Wang, W. Zhang, H. Guo, H. Bubb, and K. Ikeuchi, "A safetybased approaching behavioural model with various driving characteristics," Transportation Research C, vol. 19, no. 6, pp. 1202-1214, 2011.

[8] P. N. Seneviratne and J. F. Morrall, "Analysis of factors affecting the choice of route of pedestrians," Transportation Planning \& Technology, vol. 10, no. 2, pp. 147-159, 1985.

[9] H. Guo, W. Wang, W. Guo, X. Jiang, and H. Bubb, "Reliability analysis of pedestrian safety crossing in urban traffic environment," Safety Science, vol. 50, no. 4, pp. 968-973, 2012.

[10] A. W. J. Borgers and H. J. P. Timmermans, "City centre entry points, store location patterns and pedestrian route choice behaviour: a microlevel simulation model," Socio-Economic Planning Sciences, vol. 20, no. 1, pp. 25-31, 1986.

[11] C. Y. Cheung and W. H. K. Lam, "Pedestrian route choices between escalator and stairway in MTR stations," Journal of Transportation Engineering, vol. 124, no. 3, pp. 277-285, 1998.

[12] J. H. Ye, X. Chen, C. Yang, and J. Wu, "Walking behavior and pedestrian flow characteristics for different types of walking facilities," Transportation Research Record, vol. 2048, no. 6, pp. 43-51, 2008.

[13] I. Zeiler, C. Rudloff, and D. Bauer, "Modeling random taste variations on level changes in passenger route choice in a public transport station," in Pedestrian and Evacuation Dynamics, pp. 185-195, 2011.

[14] H.-F. Jia, B.-F. Sun, Q.-Y. Luo, and J.-C. Han, "Measurement and adaptation analysis of their facilities capacity for the subway passenger terminal," Journal of Jilin University, vol. 39, no. 2, pp. 199-203, 2009.
[15] B. Y. M. Lam and K. W. Chau, "Explaining the variations in the pedestrian flow values of shopping centres," Facilities, vol. 30, no. 3-4, pp. 164-176, 2012.

[16] E. K. Olander and F. F. Eves, "Elevator availability and its impact on stair use in a workplace," Journal of Environmental Psychology, vol. 31, no. 2, pp. 200-206, 2011.

[17] A. L. Lewis and F. F. Eves, "Testing the theory underlying the success of point-of-choice prompts: a multi-component stair climbing intervention," Psychology of Sport and Exercise, vol. 13, no. 2, pp. 126-132, 2012. 


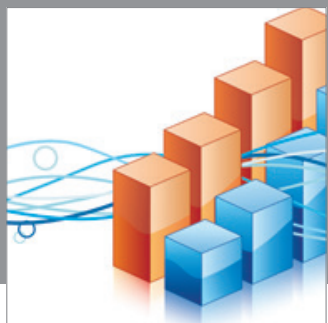

Advances in

Operations Research

mansans

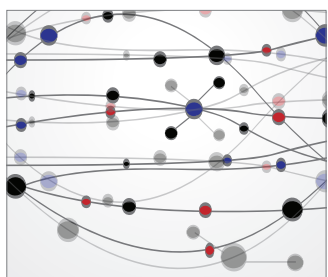

The Scientific World Journal
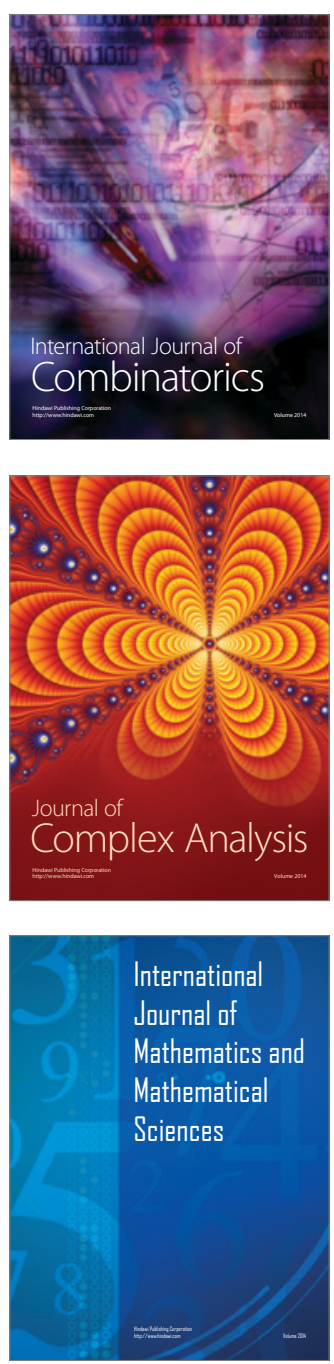
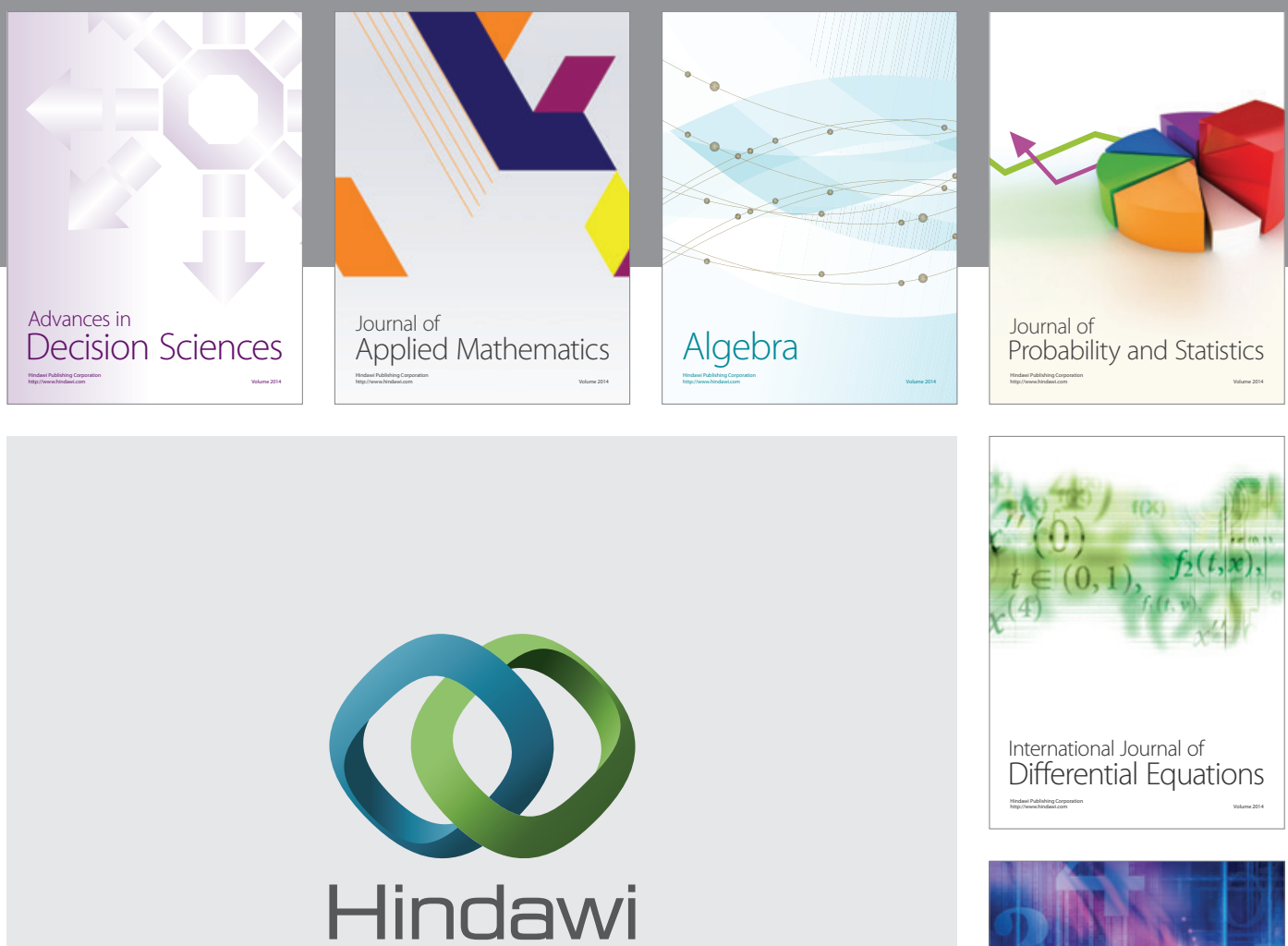

Submit your manuscripts at http://www.hindawi.com
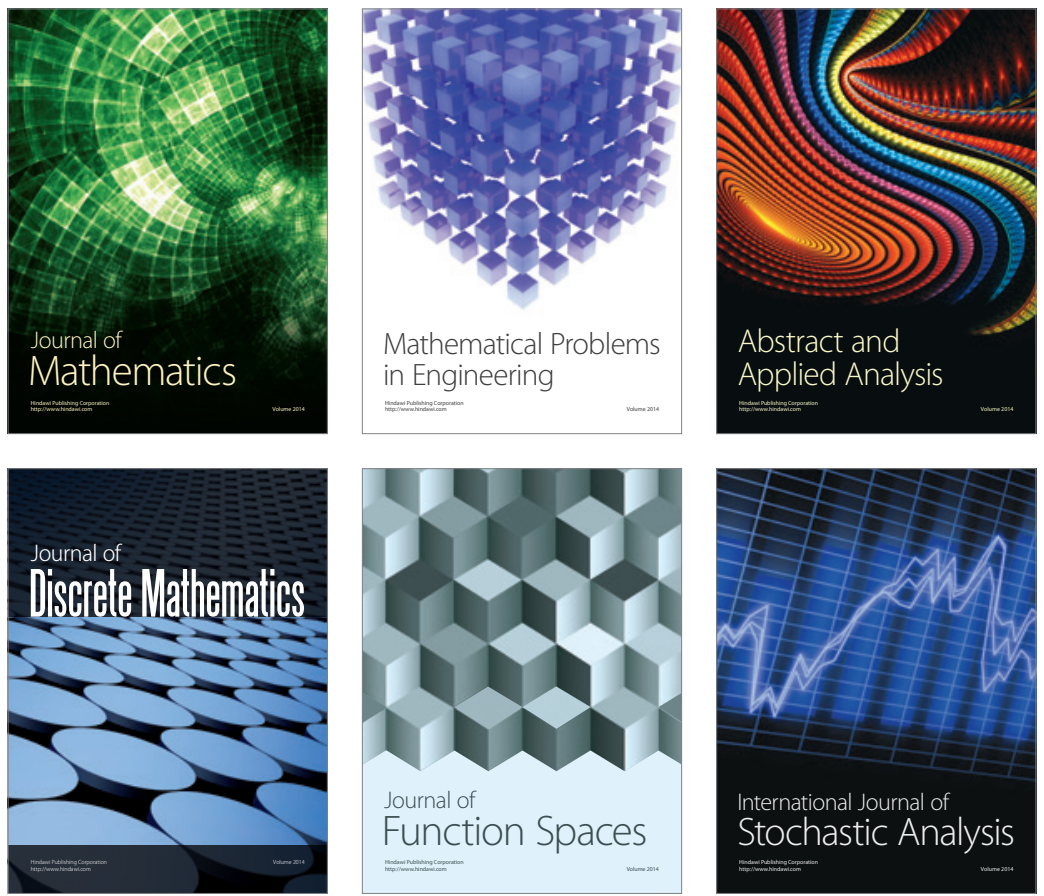

Journal of

Function Spaces

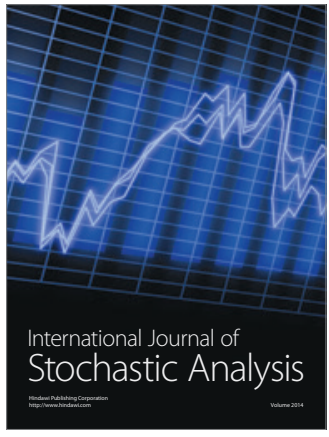

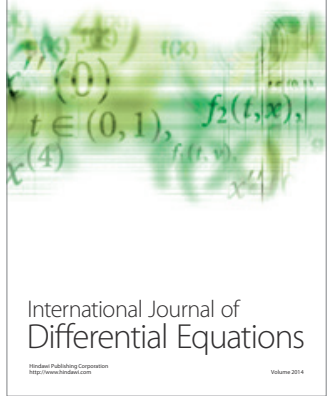
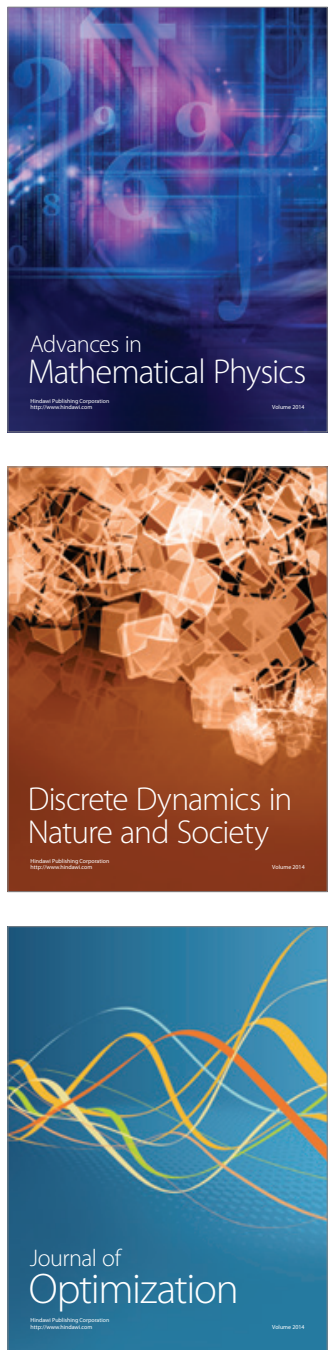